\section{[gw22-e0616] ANGIOGRAPHIC EVALUATION OF A NEW TECHNIQUE FOR COMMON FEMORAL ARTERY ACCESS: THE INGUINAL LIGAMENT-GUIDED}

Nie Shaoping, Liu Baiqiu, Jia Changqi, Zhang Yin, Lv Qiang, Liu Xinmin, Wu Jiahui, Qiao Yan, Li Jun, Luo Taiyang, Dong Jianzeng, Liu Xiaohui, Ma Changsheng Beijing Anzhen Hospital, Capital Medical University, Beijing, China

\subsection{6/heartjnl-2011-300867.469}

Objective Various techniques have been used to acquire common femoral artery (CFA) access which was proved to be safe with lower risk of complications in percutaneous catheterisation. In order to increase the success rate of CFA puncture, a relatively simple, safe and reliable technique was developed. Methods We introduced a new inguinal ligament-guided technique and inguinal division. Clinical data were collected on 822 unselected patients undergoing transfemoral percutaneous coronary catheterisation between 2004 and 2009. Inguinal ligament was utilised as a reference to conduct artery puncture site. After palpating anterior superior iliac spine and pubic symphysis with caution to locate the inguinal ligament, medius pinpointed the intersection of inguinal ligament and femoral artery. Thereafter, place forefinger beside the medius, perform artery puncture at the inferior margin of forefinger with $45^{\circ}$ modified seldinger technique. By femoral angiogram, we utilised the femoral head as a landmark, of which the midpoint, inferior and superior margin were referred to borderlines, to divide the inguinal region into zone $\mathrm{A}, \mathrm{B} 1, \mathrm{~B} 2$ and $\mathrm{C}$ so as to identify the location of femoral artery cannulation. Femoral head (zone B) was defined as the optimal area of femoral artery puncture.

Results Successful CFA cannulation occurred in $82.8 \%$ $(681 / 822)$ of cases. Arterial puncture over the femoral head 
(zone B) occurred in $96.8 \%(796 / 822)$ of patients, $36.0 \%$ (296/822) for zone B1 and 60.8\% (500/822) for zone B2, respectively. When femoral artery puncture located on zone B, B1, B2 and C, CFA cannulation occurred in $83.9 \%$ (668/796), $91.6 \%$ (271/296), 79.4\% (397/500) and 40\% (8/20), respectively.

Conclusions Inguinal ligament is a reliable reference for guiding the puncture of CFA in transfemoral percutaneous catheterisation. 